\title{
Flagellate Erythema: \\ A Case of Shiitake Dermatitis and Review of Pathogenesis
}

\author{
Authors: \\ *Kyle Wu, Sara de Menezes, Aaron Robinson \\ St Vincent's Hospital, Melbourne, Australia \\ *Correspondence to kyle.a.wu@gmail.com \\ Disclosure: $\quad$ The authors have declared no conflicts of interest. \\ Received: $\quad 27.08 .21$ \\ Accepted: $\quad 16.11 .21$ \\ Keywords: $\quad$ Allergic, dermatitis, mushroom, shiitake, toxic. \\ Citation: $\quad$ EMJ Allergy Immunol. 2022; DOI/10.33590/emjallergyimmunol/21-00187.
}

\begin{abstract}
Shiitake dermatitis is a rare eruption that is associated with the ingestion of uncooked shiitake mushrooms, resulting in a distinctive flagellate erythema. It was initially hypothesised that the mechanism of disease related to a toxic reaction to lentinan; however, recent evidence has suggested a potential allergic mechanism. The authors herein present a case of shiitake dermatitis and review the current understanding of pathogenesis of this condition and flagellate morphology. With an increase in consumption of shiitake mushrooms in Western society, shiitake dermatitis is expected to become more prevalent worldwide.
\end{abstract}

\section{INTRODUCTION}

\section{CASE REPORT}

Shiitake mushrooms (Lentinus edodes) are often used in Asian cuisine, with shiitake dermatitis first recognised in East Asian countries. Only recently has it become reported in Europe and other Western countries, correlating with a shift to more diverse cuisines worldwide.,2 Consumption of raw or undercooked shiitake mushrooms can lead to shiitake dermatitis with a distinctive erythematous eruption. This is characterised by the flagellate morphology, typically arranged in linear patterns involving the trunk and extremities. The underlying pathogenesis is currently poorly understood. It was initially hypothesised that the mechanism of disease related to a toxic reaction to lentinan; however, recent evidence has suggested a potential allergic mechanism. ${ }^{3}$ The authors herein present a case of shiitake dermatitis.

A 75-year-old male presented with a 1-day history of a mildly pruritic, linear erythematous eruption. He was otherwise systemically well. His past medical history was unremarkable, with no regular medication use. On examination, there were flagellate erythematous plaques coalescing over the abdomen and upper back, involving the neck and posterior scalp (Figures 1 and 2). There was no dermatographism, mucosal involvement, joint tenderness, proximal muscle weakness, or other examination findings of dermatomyositis. Skin biopsy showed focal spongiosis as well as superficial light perivascular chronic inflammation with rare eosinophils and neutrophils in the lumen of superficial vessels. The patient was unaware he had consumed shiitake mushrooms prior to onset of the eruption until he was shown photographs of the mushrooms. Based on his history and 


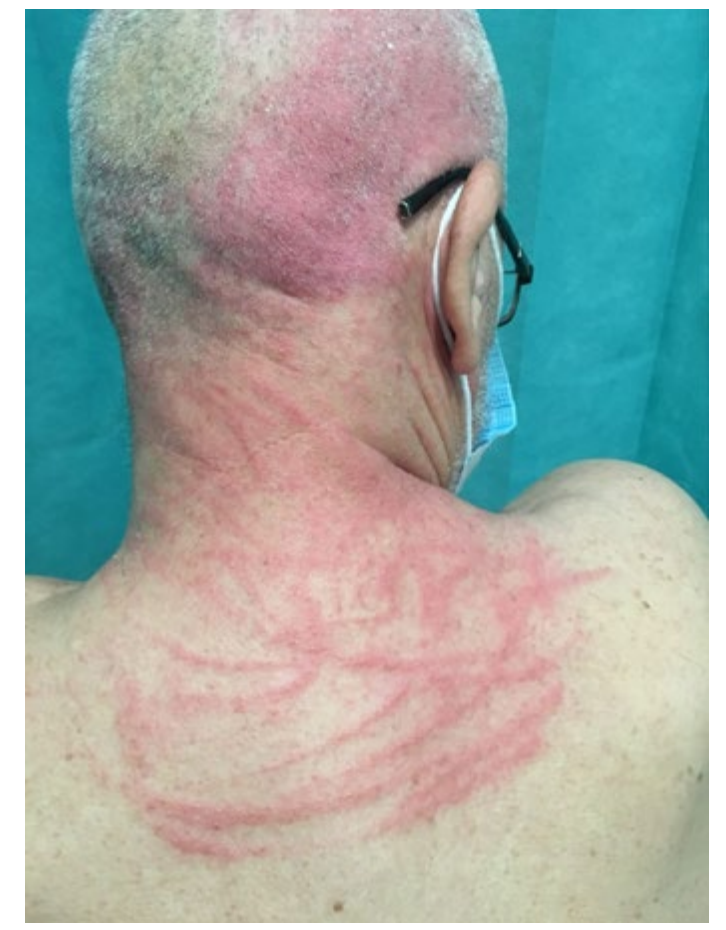

Figure 1: Linear urticarial plaques from the upper back to the posterior scalp.

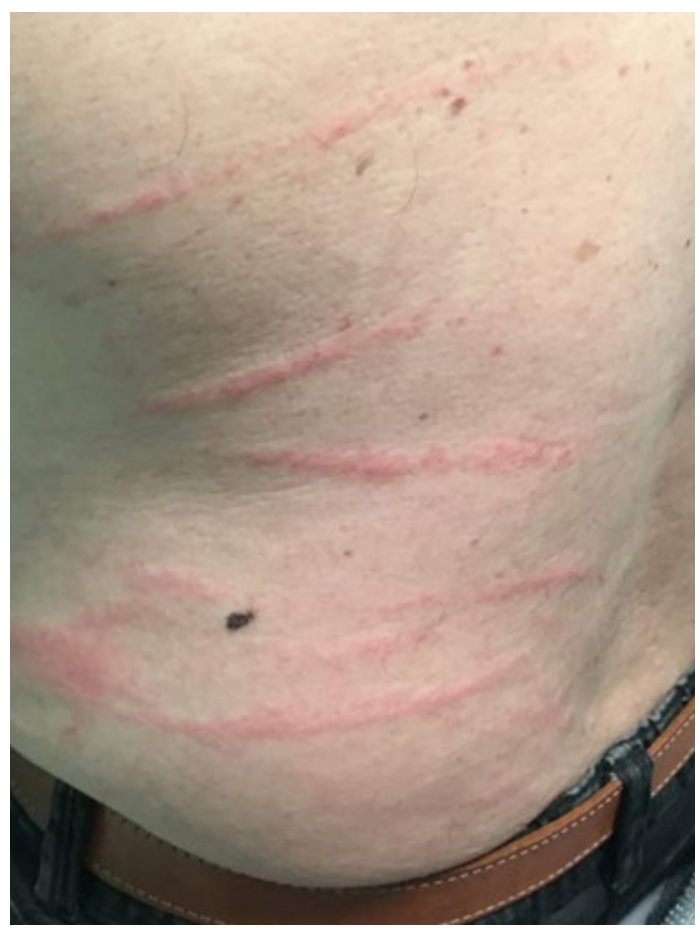

Figure 2: Linear urticarial plaques over the lower back.

clinical findings, a diagnosis of shiitake flagellate dermatitis was made. This patient was treated symptomatically with anti-histamines, topical betamethasone dipropionate ointment, and advised to avoid eating undercooked shiitake mushrooms. On follow up, the eruption had completely resolved after 10 days. 


\section{DISCUSSION}

Shiitake dermatitis is a rare and self-limiting condition that is thought to be a toxic cutaneous reaction to lentinan, a polysaccharide derived from shiitake mycelia. ${ }^{4}$ The exact pathogenesis of shiitake dermatitis and its associated flagellate erythema remains controversial. The cutaneous eruption occurs in susceptible individuals after ingesting raw or undercooked shiitake mushrooms, resulting in consumption of active lentinan. Adequate cooking of shiitake mushrooms denatures lentinan. A toxic reaction to active lentinan mediated by IL-1 secretion, causing vasodilation and haemorrhage, was initially hypothesised as the mechanism of action. ${ }^{5}$ Shiitake flagellate dermatitis was first described in Japan by Nakamura in 1977, where it was initially termed as a toxicoderma. ${ }^{6}$

Moreover, there are scant reports of patients with shiitake dermatitis having delayed positive skin prick testing, suggesting a potential delayed-type hypersensitivity mechanism to be part of the pathogenesis. ${ }^{2,3,7}$ Lentinan has also been implicated as an allergen in a few reported cases of shiitake mushroom allergic contact dermatitis as well as in cases of allergic alveolitis following inhalation of spores from the shiitake mushroom. ${ }^{7-9}$ The relatively low incidence of shiitake mushroom dermatitis in contrast to its widespread consumption as well as a lack of outbreaks in groups of people with exposure to shared mushrooms may be explained by individual susceptibilities or hypersensitivity rather than a solely toxic reaction to active lentinan.

The mechanism behind the distinctive flagellate or 'whip-like' morphology also remains unclear. The degree of pruritus is variable amongst reported cases, with often a delay between time of insult and lesion onset. While it has been attributed to Koebner phenomenon induced by scratch injury, it has been reported that flagellate lesions are not immediately elicited after scratching. It is proposed that minimal trauma during periods of high serum lentinan leads to local deposition and upregulation of relevant inflammatory mediators and neuropeptides. ${ }^{10,11}$ It has also been postulated that photosensitisation by active lentinan may underlie the mechanism of disease; however, in the authors' case, the cutaneous eruption was not limited to sun-exposed areas.

The offending agent and potential allergen, lentinan, has been investigated for potential anti-tumour properties through enhancement of the host immune and complement systems. A Japanese study found that patients with gastric cancer receiving lentinan in combination with paclitaxel or cisplatin chemotherapy resulted in longer overall median survival compared to patients receiving chemotherapy alone.12 Ongoing large-scale studies are required to better evaluate the biological properties of lentinan in modulation of the immune system.

Shiitake dermatitis is usually self-limited, resolving in 2-4 weeks with symptomatic pruritic management, including emollients, topical steroids, and antihistamines. Adjunctive photography during patient assessment may be useful to confirm the diagnosis and history of shitake mushroom consumption. Skin biopsy findings of shiitake dermatitis are non-specific, largely demonstrating spongiosis, papillary dermis oedema, and perivascular inflammatory infiltrate without vasculitis. Shiitake dermatitis, therefore, remains a primarily clinical diagnosis. Differentials for the characteristic eruption include bleomycin-induced flagellate dermatitis, dermatomyositis, and adult-onset Still's disease."

\section{CONCLUSION}

Given the growing popularity and availability of diverse foods, cases of shiitake dermatitis may be expected to become more prevalent, and it is, therefore, important to recognise this condition in patients presenting with this clinical picture. The underlying mechanism of shiitake dermatitis and the immune reaction to lentinan remain to be fully understood. 


\section{References}

1. Boels D et al. Shiitake dermatitis recorded by French Poison Control Centers - new case series with clinical observations. Clin Toxicol (Phila). 2014;52(6):625-8.

2. Netchiporouk E et al. Pustular flagellate dermatitis after consumption of shiitake mushrooms. JAAD Case Rep. 2015;1(3):117-9.

3. Corazza M et al. Shiitake dermatitis: toxic or allergic reaction? J Eur Acad Dermatol Venereol. 2015;29(7):144951.

4. Forward E et al. A case of shiitake mushroom dermatitis. Australas J
Dermatol. 2018;59(4):337-8.

5. Mendonça $\mathrm{C}$ et al. Shiitake dermatitis An Bras Dermatol. 2015;90(2):276-8.

6. Nakamura T. Toxicoderma caused by shiitake (Lentinus edodes). Jpn J Clin Dermatol. 1977;31(2):65-8.

7. Tarvainen $\mathrm{K}$ et al. Allergy and toxicodermia from shiitake mushrooms. J Am Acad Dermatol. 1991;24(1):64-6.

8. Curnow P, Tam M. Contact dermatitis to shiitake mushroom. Australas J Dermatol. 2003;44(2):155-7.

9. Kopp $\mathrm{T}$ et al. Systemic allergic contact dermatitis due to consumption of raw shiitake mushroom. Clin Exp Dermatol. 2009;34(8):e910-3.

10. Ade R et al. Shiitake dermatitis demonstrating Köebner phenomenon Int J Dermatol. 2015;54(5):e179-81.

11. Chu EY et al. Shiitake dermatitis: a report of 3 cases and review of the literature. Cutis. 2013;91(6):287-90.

12. Ina $\mathrm{K}$ et al. Lentinan prolonged survival in patients with gastric cancer receiving S-1-based chemotherapy. World J Clin Oncol. 2011:2(10):339-43. 